PROCEEDINGS OF THE

AMERICAN MATHEMATICAL SOCIETY

Volume 139, Number 12, December 2011, Pages 4331-4342

S 0002-9939(2011)10875-X

Article electronically published on April 11, 2011

\title{
SPECTRAL PROPERTIES OF COMMUTING OPERATIONS FOR $n$-TUPLES
}

\author{
C. BENHIDA AND E. H. ZEROUALI
}

(Communicated by Marius Junge)

\begin{abstract}
Let $\mathbf{R}$ and $\mathbf{S}$ be commuting $n$-tuples. We give some spectral and local spectral relations between $\mathbf{R S}$ and $\mathbf{S R}$. In particular, we show that $\mathbf{R S}$ has the single valued extension property or satisfies Bishop's property $(\beta)$ if and only if SR has the corresponding property.
\end{abstract}

\section{INTRODUCTION}

Let $X$ be a Banach space and let $\mathcal{L}(X)$ denote the Banach algebra of all bounded linear operators on $X$. Given two bounded operators $R$ and $S$ defined on $X$, Jacobson's lemma says

$$
\sigma(R S) \backslash\{0\}=\sigma(S R) \backslash\{0\} .
$$

Several works have been devoted to the previous equation, which has been extended as follows:

$$
\Sigma(R S) \backslash\{0\}=\Sigma(S R) \backslash\{0\},
$$

where $\Sigma$ runs over a large class of spectra. See [2, 3, for example.

We showed in [3] that $R S$ and $S R$ also share most of their local spectral properties. In particular, If $R S$ has the single valued extension property $(S V E P)$ (resp. Bishop's property $(\beta)$ ), then $S R$ has $(S V E P)$ (resp. Bishop's property $(\beta)$ ).

Jacobson's lemma does not extend to pairs of systems of two or more variables: for example if $U V=I \neq V U$, then it fails for the pair $(\mathbf{R}, \mathbf{S})=\left(\frac{1}{2} I, \frac{1}{2} V\right),(I-$ $U V, U)$ ). With enough mutual commutativity however, analogues of (10) can be extended to a product system RS.

Recall that an operator $\mathbf{T}=\left(T_{1}, \cdots, T_{n}\right) \in \mathcal{L}(X)^{n}$ is called a commuting $n$ tuple if $T_{i} T_{j}=T_{j} T_{i}$ for every $1 \leq i, j \leq n$. The notion of spectrum for commuting $n$-tuples was introduced by J. L. Taylor in [13]; see also [17. Various spectra have been adapted to this situation using Taylor's formalism.

The main goal of this paper is to develop analogues of the preceding results in the setting of $n$-tuples where all spectra are considered in the sense of Taylor. To this aim, let $\mathbf{R}=\left(R_{1}, \cdots, R_{n}\right)$ and $\mathbf{S}=\left(S_{1}, \cdots, S_{n}\right)$ be commuting $n$-tuples and consider $\mathbf{R S}=:\left(R_{1} S_{1}, \cdots, R_{n} S_{n}\right)$. To ensure that $\mathbf{R S}$ remains a commuting

Received by the editors February 16, 2009 and, in revised form, January 31, 2010 and October 6,2010 .

2010 Mathematics Subject Classification. Primary 47B20.

Key words and phrases. $n$-tuples, Taylor spectrum, joint spectra, SVEP, Bishop's property $(\beta)$. 
$n$-tuple, suitable extra conditions are needed. The first condition is the so-called "criss-cross commutativity" and has been intensively studied in the literature.

Recall that the commuting $n$-tuples $\mathbf{R}=\left(R_{1}, \cdots, R_{n}\right)$ and $\mathbf{S}=\left(S_{1}, \cdots, S_{n}\right)$ are said to be criss-cross commuting provided that

$$
R_{i} S_{j} R_{k}=R_{k} S_{j} R_{i} \text { and } S_{i} R_{j} S_{k}=S_{k} R_{j} S_{i} \text { for every } 1 \leq i, j, k \leq n .
$$

It follows that $\mathbf{S R}$ and $\mathbf{R S}$ are commuting $n$-tuples [9, 10, and then equation (1) is obtained for several Taylor spectra. See [5] and [11.

The second commutativity condition is given in [4. It is assumed that $\mathbf{R}$ and $\mathbf{S}$ are commuting $n$-tuples. We will say that $\mathbf{R}$ and $\mathbf{S}$ are nearly commuting provided that

$$
R_{i} S_{j}=S_{j} R_{i} \quad \text { for every } i \neq j .
$$

Again, under near commutativity, SR and $\mathbf{R S}$ are commuting $n$-tuples, and they share their spectral properties except in

$$
[0]:=\left\{\left(z_{1}, \cdots, z_{n}\right): \prod_{i=1}^{n} z_{i}=0\right\}
$$

More precisely,

$$
\Sigma(R S) \backslash[0]=\Sigma(S R) \backslash[0],
$$

where $\Sigma$ runs over a large class of Taylor spectra.

Remark 1. Before proceeding further, we mention as observed in [3] that near commutativity and criss-cross commutativity are distinct. In [3], examples of $n$ tuples satisfying only near commutativity and criss-cross commutativity are given.

We pursue in this paper our investigations into common spectral properties of the $n$-tuples RS and SR under any of the previous commutativity conditions. In section 2 we introduce basic definitions of Taylor spectrum and give the corresponding local spectral properties. In section 3 we retrieve many results on common spectral properties of RS and SR. The given proofs unify most of the earliest results in this context. Section 4 is devoted to local spectral theory for commuting $n$-tuples. We show that if RS has $S V E P$ or Bishop's property $(\beta)$, then so has $\mathbf{S R}$. We end in section 5 with some results involving local spectra of $\mathbf{R S}$ and $\mathbf{S R}$.

\section{BASIC DEFINITIONS}

Let $\mathbf{e}=\left\{e_{1}, e_{2}, \cdots, e_{n}\right\}$ be indeterminate and define $\Lambda_{n}[\mathbf{e}]$ to be the exterior algebra on the generators $e_{1}, e_{2}, \ldots, e_{n}$, that is, the linear space over the complex plane $\mathbb{C}$ endowed with an anti-commutative exterior product $e_{i} \wedge e_{j}=-e_{j} \wedge e_{i}(1 \leq$ $i, j \leq n)$. For $F=\left(i_{1}, \cdots, i_{p}\right)\{F\}=\left\{i_{1}, \cdots, i_{p}\right\} \subset\{1, \cdots, n\}$ with $i_{1}<\cdots<i_{p}$, we write $e_{F}=e_{i_{1}} \wedge \cdots \wedge e_{i_{p}}$. The exterior algebra over $\mathbb{C}$ is then given by

$$
\Lambda_{n}[\mathbf{e}]=\left\{\sum_{F} \alpha_{F} e_{F}: e_{F}=e_{i_{1}} \wedge \cdots \wedge e_{i_{p}} \text { and } \alpha_{F} \in \mathbb{C}\right\} .
$$

The identity element for the exterior product will be denoted by $e_{\emptyset}$. Consider $\Lambda_{n}^{k}[\mathbf{e}]=:\left\{\sum_{|F|=k} \alpha_{F} e_{F}: \alpha_{F} \in \mathbb{C}\right\}$, where $|F|$ is the cardinal of $\{F\}$. Then clearly $\operatorname{dim} \Lambda_{n}^{k}[\mathbf{e}]=C_{n}^{k}$ for every $k \leq n, \Lambda_{n}^{k}[\mathbf{e}] \wedge \Lambda_{n}^{l}[\mathbf{e}]=\Lambda_{n}^{k+l}[\mathbf{e}]$ and $\Lambda_{n}[\mathbf{e}]=\bigoplus_{k=0}^{n} \Lambda_{n}^{k}$. 
Given a Banach space $X$, the exterior algebra over $X$ is defined to be

$$
\Lambda_{n}[\mathbf{e}, X]=\left\{\sum_{F} x_{F} e_{F}: e_{F}=e_{i_{1}} \wedge \cdots \wedge e_{i_{p}} \text { and } x_{F} \in X\right\} .
$$

The subspaces $\Lambda_{n}^{p}[\mathbf{e}, X]=\left\{\sum_{|F|=p} x_{F} e_{F}: x_{F} \in X\right\}$ are given for $p \leq n$ in a similar way. Naturally $\Lambda_{n}^{0}[\mathbf{e}, X], \Lambda_{n}^{1}[\mathbf{e}, X]$ and $\Lambda_{n}^{n}[\mathbf{e}, X]$ can be identified with $X, X^{n}$ and $X$ respectively.

Since no confusion is possible we will omit any reference to the indeterminate $e_{1}, e_{2}, \cdots, e_{n}$, and for short we will write $\Lambda_{n}^{k}[X]$ and $\Lambda_{n}[X]$ for $\Lambda_{n}^{k}[\mathbf{e}, X]$ and $\Lambda_{n}[\mathbf{e}, X]$ respectively.

For $S \in \mathcal{L}(X)$, the same symbol $S$ is usually kept to denote the extended operator defined on $\Lambda_{n}[X]$ by

$$
\Lambda_{n}(S): S\left(\sum_{F} x_{F} e_{F}\right)=\sum_{F} S x_{F} e_{F} .
$$

Given $i \in\{1,2, \cdots, n\}$, let $E_{i}: \Lambda_{n}[X] \rightarrow \Lambda_{n}[X], E_{i}\left(e_{F}\right)=e_{i} \wedge e_{F}$ be the left multiplication operator induced by $e_{i}$. With any commuting $n$-tuple $\mathbf{T}=$ $\left(T_{1}, \cdots, T_{n}\right)$ we associate the linear mapping defined over $\Lambda_{n}[X]$ by

$$
\alpha_{\mathbf{T}}=\sum_{i=1}^{n} T_{i} \otimes E_{i}: \sum_{F} x_{F} e_{F} \rightarrow \sum_{F} \sum_{i=1}^{n} T_{i} x_{F} e_{i} \wedge e_{F}
$$

Clearly,

$$
\left(\alpha_{\mathbf{T}}\right)^{2} x_{F} e_{F}=\sum_{i, j=1}^{n} T_{j} T_{i} x_{F} e_{j} \wedge e_{i} \wedge e_{F}=\sum_{i<j} T_{i} T_{j} x_{F}\left(e_{i} \wedge e_{j}+e_{j} \wedge e_{i}\right) \wedge e_{F}=0 .
$$

It follows that $\left(\alpha_{\mathbf{T}}\right)^{2}=0$ and hence that $\operatorname{Im}\left(\alpha_{\mathbf{T}}\right) \subset \operatorname{ker}\left(\alpha_{\mathbf{T}}\right)$. Since $\alpha_{\mathbf{T}}$ takes $\Lambda_{n}^{k-1}[X]$ to $\Lambda_{n}^{k}[X]$, the last statement is equivalent to: $\operatorname{Im}\left(\left.\alpha_{T}\right|_{\Lambda_{n}^{k-1}[X]}\right) \subset$ $\operatorname{ker}\left(\alpha_{\mathbf{T} \mid \Lambda_{n}^{k}[X]}\right)$ for every $k \leq n$.

Set $\alpha_{\mathbf{T}}^{k}:=\left.\alpha_{\mathbf{T}}\right|_{\Lambda_{n}^{k}[X]}$. We construct a co-chain complex $K(\mathbf{T}, X)$, called the Koszul complex associated with $\mathbf{T}$ on $X$, as follows:

$$
K(\mathbf{T}, X): \quad \mathbf{0} \stackrel{\alpha_{\mathbf{T}}^{-1}}{\longrightarrow} \Lambda_{n}^{0}[X] \stackrel{\alpha_{\mathbf{T}}^{0}}{\longrightarrow} \Lambda_{n}^{1}[X] \stackrel{\alpha_{\mathbf{T}}^{1}}{\longrightarrow} \ldots \stackrel{\alpha_{\mathbf{T}}^{n-1}}{\longrightarrow} \Lambda_{n}^{n}[X] \stackrel{\alpha_{\mathbf{T}}^{n}}{\longrightarrow} \mathbf{0} .
$$

The operator $\mathbf{T}$ is said to be non-singular, or Taylor invertible, if $\operatorname{ker}\left(\alpha_{\mathbf{T}}^{k}\right)=$ $\operatorname{Im}\left(\alpha_{\mathbf{T}}^{k-1}\right)$ for $k=0, \cdots, n$. The associated Koszul complex is said to be exact in this case. The Taylor spectrum of $\mathbf{T}$ on $X^{n}$ is then the set

$$
\sigma_{T}(\mathbf{T})=\left\{\lambda \in \mathbb{C}^{n}: K(\mathbf{T}-\lambda, X) \text { is not exact }\right\} .
$$

The Taylor spectrum coincides with the usual spectrum in the case of single operators. We refer to [13, 15, 16, 17, for a detailed study of these spectra.

These notions are taken to sheaf theory canonically to define the corresponding local spectral properties. For $\mathcal{U}$ an open set in $\mathbb{C}^{n}$, denote by $\mathcal{O}(\mathcal{U}, X)$ the Fréchet algebra of analytic functions on $\mathcal{U}$. We define

$K .(\mathbf{T}-\mathbf{z}): \mathbf{0} \stackrel{\alpha_{\mathbf{T}-\mathbf{z}}^{-1}}{\longrightarrow} \Lambda_{n}^{0}[\mathcal{O}(\mathcal{U}, X)] \stackrel{\alpha_{\mathbf{T}-\mathbf{z}}^{0}}{\longrightarrow} \Lambda_{n}^{1}[\mathcal{O}(\mathcal{U}, X)] \stackrel{\alpha_{\mathbf{T}-\mathbf{z}}^{1}}{\longrightarrow} \cdots \stackrel{\alpha_{\mathbf{T}-\mathbf{z}}^{n-1}}{\longrightarrow} \Lambda_{n}^{n}[\mathcal{O}(\mathcal{U}, X)] \stackrel{\alpha_{\mathbf{T}-\mathbf{z}}^{n}}{\longrightarrow} \mathbf{0}$, and we consider the associated cohomology groups

$$
H^{k}(\mathbf{T}-\mathbf{z}, \mathcal{O}(\mathcal{U}, X))=\operatorname{ker}\left(\alpha_{\mathbf{T}-\mathbf{z}}^{k}\right) / \operatorname{Im}\left(\alpha_{\mathbf{T}-\mathbf{z}}^{k-1}\right), \quad k=1, \cdots, n .
$$


Let $\mathbf{T}$ be a commuting $n$-tuple. We will say that $\mathbf{T}$ has the $S V E P$ at $\lambda$ if there exists an open polydisc $D_{\lambda}$ centered at $\lambda$ such that $H^{k}\left(\mathcal{O}\left(D_{\lambda}, X\right), \mathbf{T}\right)=0$ for $k=1, \cdots, n-1$.

Following the definition given by J. Eschmeier [6, a commuting $n$-tuple $\mathbf{T}$ has $S V E P$ provided that the cohomology groups $H^{k}(\mathcal{O}(\mathcal{U}(z), X))=\{0\}$ for $k=$ $1, \cdots, n-1$ and for every $z \in \mathbb{C}$. As usual $\mathcal{O}(\mathcal{U}(z), X)$ stands for the space of all $X$-valued analytic functions near $\{z\}$. It is then not difficult to show that $\mathbf{T}$ has $S V E P$ in the sense of $[\underline{6}$ if and only if $\mathbf{T}$ has $S V E P$, at every $\lambda \in \mathbb{C}$.

A detailed study of the $S V E P$ for commuting $n$-tuples is developed in [6]. It is in particular shown that if $\left(T_{1}, \cdots, T_{n}\right)$ has $S V E P$, then $\left(T_{1}, \cdots, T_{n-1}\right)$ has $S V E P$, and the same for $\left(T_{\sigma(1)}, \cdots, T_{\sigma(n)}\right)$ for every permutation $\sigma$ of $\{1, \cdots, n\}$.

Other notions including the $(\beta)$ condition and spectral decomposition are defined in a similar way. We refer to [7, 16] for more details.

\section{Common spectral properties between RS and SR}

Using a nice lemma from [7], we retrieve in a simple way several results given in 4, 9, 10, 11. We thank J. Eschmeier for fruitful discussions and for pointing out to us this next lemma.

Lemma 1 ([7, Lemma 2.2.4]). Let $a=\left(a_{1}, \cdots, a_{n}\right)$ be a commuting n-tuple of morphisms of module $X$. Let $b_{1}, \cdots, b_{n}$ belong to the commutant of $a$ and consider $c=a_{1} b_{1}+\cdots+a_{n} b_{n}$. Then $M_{c}: H^{p}(a, X) \longrightarrow H^{p}(a, X)$, induced by the componentwise action of $c$, is the zero operator for each $p$.

3.1. Common spectral properties under near commutativity. Let $\mathbf{R}=$ $\left(R_{1}, \cdots, R_{n}\right) \in \mathcal{L}(X)$ and $\mathbf{S}=\left(S_{1}, \cdots, S_{n}\right) \in \mathcal{L}(X)$ be commuting $n$-tuples that are nearly commuting. We fix $i \in\{1, \cdots, n\}$ and we set $\mathbf{S R}=\left(S_{1} R_{1}, \cdots, S_{i} R_{i}, \cdots\right.$, $\left.S_{n} R_{n}\right)$ and $(\mathbf{S R})_{\mathbf{i}}=\left(S_{1} R_{1}, \cdots, R_{i} S_{i}, \cdots, S_{n} R_{n}\right)$.

Let $z \in \mathbb{C}^{n}$ be such that $z_{i} \neq 0$. The componentwise actions

$$
\phi: u \in \Lambda^{p}[X] \mapsto R_{i} u \in \Lambda^{p}[X] \text { and } \psi: u \in \Lambda^{p}[X] \mapsto S_{i} u \in \Lambda^{p}[X]
$$

define morphisms of complexes

$$
\begin{aligned}
& K(\mathbf{S R}-\mathbf{z}, X) \stackrel{\phi}{\longrightarrow} K\left((\mathbf{S R})_{\mathbf{i}}-\mathbf{z}, X\right), \\
& K\left((\mathbf{S R})_{\mathbf{i}}-\mathbf{z}, X\right) \stackrel{\psi}{\longrightarrow} K(\mathbf{S R}-\mathbf{z}, X) .
\end{aligned}
$$

The mapping $z_{i} I-\psi \phi$ is then given by the componentwise action and satisfies $z_{i} I-S_{i} R_{i}=\left(z_{i}-S_{i} R_{i}\right)=\sum_{v=1}^{n}\left(z_{v}-S_{v} R_{v}\right) C_{v}$, where $C_{v}=\delta_{i v} I \in\{\mathbf{S R}\}^{\prime}$ for $v=1, \cdots, n$ (here $\{\mathbf{S R}\}^{\prime}$ denotes the commutant of $\mathbf{S R}$ ). It follows from Lemma 1 that $\psi \phi=z_{i} I$ on $H^{p}(\mathbf{S R}-\mathbf{z}, X)$ for $p \in\{0, \cdots, n\}$. Similarly, from the identity $z_{i} I-R_{i} S_{i}=\left(z_{i}-R_{i} S_{i}\right)=\sum_{v=1}^{n}\left(z_{v}-R_{v} S_{v}\right) C_{v}^{\prime}$, where $C_{v}^{\prime}=\delta_{i v} I \in\left\{(\mathbf{S R})_{\mathbf{i}}\right\}^{\prime}$ for $v=1, \cdots, n$, we get $\phi \psi=z_{i} 1$ on $H^{p}\left((\mathbf{S R})_{\mathbf{i}}-\mathbf{z}, X\right)$ for $p \in\{0, \cdots, n\}$.

Thus,

$$
\begin{array}{ccc}
\phi: H^{p}(\mathbf{S R}-\mathbf{z}, X) & \longrightarrow & H^{p}\left((\mathbf{S R})_{\mathbf{i}}-\mathbf{z}, X\right), \\
\psi: H^{p}\left((\mathbf{S R})_{\mathbf{i}}-\mathbf{z}, X\right) & \longrightarrow & H^{p}(\mathbf{S R}-\mathbf{z}, X)
\end{array}
$$

are vector-space bounded isomorphisms, for every $p=0, \cdots, n$, which are inverse to each other (because $z_{i} \neq 0$ ). 
As an immediate consequence from our preceding discussion, we observe that $H^{p}(\mathbf{S R}-\mathbf{z}, X)=\{0\}$ (resp. is closed) if and only if $H^{p}\left((\mathbf{S R})_{\mathbf{i}}-\mathbf{z}, X\right)=\{0\}$ (resp. is closed). Using the definitions and notation from [7, chapter 2.6], we get

Proposition 1. Let $\mathbf{S R}$ and $(\mathbf{S R})_{\mathbf{i}}$ be as above, $z \in \mathbb{C}^{n}$ be such that $z_{i} \neq 0$ and $k \in\{0, \cdots, n\}$. Then:

(1) $z \in \sigma(\mathbf{S R}) \Longleftrightarrow z \in \sigma\left((\mathbf{S R})_{\mathbf{i}}\right)$,

(2) $z \in \sigma_{e}(\mathbf{S R}) \Longleftrightarrow z \in \sigma_{e}\left((\mathbf{S R})_{\mathbf{i}}\right)$,

(3) $z \in \sigma^{\delta, k}(\mathbf{S R}) \Longleftrightarrow z \in \sigma^{\delta, k}\left((\mathbf{S R})_{\mathbf{i}}\right)$,

(4) $z \in \sigma_{e}^{\delta, k}(\mathbf{S R}) \Longleftrightarrow z \in \sigma_{e}^{\delta, k}\left((\mathbf{S R})_{\mathbf{i}}\right)$,

(5) $z \in \sigma^{\pi, k}(\mathbf{S R}) \Longleftrightarrow z \in \sigma^{\pi, k}\left((\mathbf{S R})_{\mathbf{i}}\right)$,

(6) $z \in \sigma_{e}^{\pi, k}(\mathbf{S R}) \Longleftrightarrow z \in \sigma_{e}^{\pi, k}\left((\mathbf{S R})_{\mathbf{i}}\right)$.

Let $\mathcal{I}$ be a subset of $\{1, \cdots, n\}$ and denote $(\mathbf{R S})_{\mathcal{I}}=\left(\left(R_{1} S_{1}\right)_{\mathcal{I}}, \cdots,\left(R_{n} S_{n}\right)_{\mathcal{I}}\right)$, with $\left(R_{i} S_{i}\right)_{\mathcal{I}}=S_{i} R_{i}$ if $i \in \mathcal{I}$ and $\left(R_{i} S_{i}\right)_{\mathcal{I}}=R_{i} S_{i}$ otherwise. Clearly $\left((\mathbf{R S})_{\mathcal{I}}\right)_{\mathcal{I}}=$ $\mathbf{R S}$ and $\mathbf{S R}=(\mathbf{R S})_{\{1, \cdots, n\}}$. We also write

$$
[0]^{\mathcal{I}}=\left\{\left(\lambda_{1}, \cdots, \lambda_{n}\right) \in[0]: \prod_{i \in \mathcal{I}} \lambda_{i} \neq 0\right\} .
$$

Now inductive reasoning in the previous proposition leads obviously to the next theorem.

Theorem 1. Let $\mathbf{R}=\left(R_{1}, \cdots, R_{n}\right) \in \mathcal{L}(X)$ and $\mathbf{S}=\left(S_{1}, \cdots, S_{n}\right) \in \mathcal{L}(X)$ be commuting $n$-tuples nearly commuting.

- For $\mathcal{I}, J \subset\{1, \cdots, n\}$, we have

$$
\Sigma\left((\mathbf{R S})_{\mathcal{I}}\right) \backslash[0]^{\mathcal{I} \cup \mathcal{J}}=\Sigma\left((\mathbf{S R})_{\mathcal{J}}\right) \backslash[0]^{\mathcal{I} \cup \mathcal{J}} .
$$

- In particular,

$$
\begin{aligned}
& \Sigma(\mathbf{R S}) \backslash[0]=\Sigma(\mathbf{S R}) \backslash[0] \\
& \text { for } \Sigma \in\left\{\sigma, \sigma_{e}, \sigma^{\pi, k}, \sigma_{e}^{\pi, k}, \sigma^{\delta, k}, \sigma_{e}^{\delta, k}\right\} .
\end{aligned}
$$

3.2. Common spectral properties under the criss-cross commutativity.

Let $\mathbf{R}$ and $\mathbf{S}$ be criss-cross commuting $n$-tuples. It is not difficult to check that $S_{i} \alpha_{\mathbf{R S}-\mathbf{z}}=\alpha_{\mathbf{S R}-\mathbf{z}} S_{i}$ for every $i=1, \cdots, n$. It will follow as in the first part of this section that for every $\mathbf{z} \neq(0, \cdots, 0)$, the Koszul complexes $K(\mathbf{R S}-\mathbf{z}, X)$ and $K(\mathbf{S R}-\mathbf{z}, X)$ are isomorphic. Precisely, we write the same arguments with $i$ such that $z_{i} \neq 0$. This yields to the next criss-cross version of Theorem 1

Theorem 2. Let $\mathbf{R}=\left(R_{1}, \cdots, R_{n}\right) \in \mathcal{L}(X)$ and $\mathbf{S}=\left(S_{1}, \cdots, S_{n}\right) \in \mathcal{L}(X)$ be commuting $n$-tuples satisfying the criss-cross commutativity condition. We have

$$
\Sigma(\mathbf{R S}) \backslash\{0\}=\Sigma(\mathbf{S R}) \backslash\{0\}
$$

for any $\Sigma \in\left\{\sigma, \sigma_{e}, \sigma^{\pi, k}, \sigma_{e}^{\pi, k}, \sigma^{\delta, k}, \sigma_{e}^{\delta, k}\right\}$ and $0 \leq k \leq n$.

Theorem 2 provides a simple proof for various joint spectra and generalizes the results in [9, 10. See also [1]. 


\section{Single valued extension property and Bishop's Property $(\beta)$ FOR SR AND RS}

Recall that a single operator $T$ satisfies the $S V E P$ at $\lambda \in \mathbb{C}$ if there exists a neighborhood $\mathcal{U}$ of $\lambda$ such that the zero function is the only analytic function $f$ defined on $\mathcal{U}$ satisfying $(T-\mu) f(\mu)=0$ for every $\mu \in \mathcal{U}$. The definition above can be viewed as a particular case of the $n$-tuples one (see [1]).

A result of Finch in 8 asserts that for an operator $T \in \mathcal{L}(X)$ with $S V E P$ at zero we have: $T$ is invertible if and only if $T(X)=X$. The following extension of Finch's result for $n$-tuples has been observed in [17].

Proposition 2. Let $\mathbf{T}$ be a commuting n-tuple with SVEP at zero. Then, $\mathbf{T}$ is Taylor invertible if and only if $T_{1}(X)+T_{2}(X)+\cdots+T_{n}(X)=X$.

Remark that the condition $T_{1}(X)+T_{2}(X)+\cdots+T_{n}(X)=X$ implies that $H^{n}(T)=\{0\}$, and from the proof of lemma 2.1 in [13], it follows that $H^{n}(\mathbf{T}-\lambda)=$ $\{0\}$ for $\lambda \in D$, with $D$ a polydisc containing zero. We deduce that $H^{n}(\mathcal{O}(\mathcal{U}(z), X))$ $=0$ and hence that $\mathbf{T}$ is Taylor invertible.

The commuting $n$-tuple $\mathbf{T}$ is said to possess Bishop's property $(\beta)$ if the Koszul complex $K .(T-z, \mathcal{O}(U, X))$ has a separated cohomology in degree $n$ and it is exact in degree less than $n$ for every open set $\mathcal{U} \subset \mathbb{C}^{n}$. Equivalently, $\mathbf{T}$ possesses $(\beta)$ if and only if it has $S V E P$, and $\alpha_{\mathbf{T}-\mathbf{z}}^{n-1}(\mathcal{O}(\mathcal{U}, X))$ is closed for every open set $\mathcal{U} \subset \mathbb{C}^{n}$. The last assertion can be rephrased as follows: the canonical mapping

$$
\Lambda^{n-1}[\mathcal{O}(\mathcal{U}, X)] / \operatorname{ker}\left(\alpha_{\mathbf{T}-\mathbf{z}}^{n-1}\right) \longrightarrow \operatorname{Im}\left(\alpha_{\mathbf{T}-\mathbf{z}}^{n-1}\right)
$$

is bounded below for every open set $\mathcal{U} \subset \mathbb{C}^{n}$.

Moreover, outlining the same proof for $S V E P$, one may deduce that if $\left(T_{1}, \cdots, T_{n}\right)$ possesses Bishop's property $(\beta)$, then $\left(T_{1}, \cdots, T_{n-1}\right)$ possesses Bishop's property $(\beta)$ and also $\left(T_{\sigma(1)}, \cdots, T_{\sigma(n)}\right)$ for every permutation $\sigma$.

Of course, this also generalizes the definition of Bishop's property $(\beta)$ for a single operator, which asserts that the map

$$
T-z: \mathcal{O}(\mathcal{U}, X) \longrightarrow \mathcal{O}(\mathcal{U}, X)
$$

is one to one with closed range for every open set $\mathcal{U} \subset \mathbb{C}$.

\subsection{The case of near commutativity.}

Theorem 3. Let $\mathbf{R}$ and $\mathbf{S}$ be commuting $n$-tuples nearly commuting. Then $\mathbf{T}=$ $\left(R_{1} S_{1}, \cdots, R_{i} S_{i}, \cdots, R_{n} S_{n}\right)$ has $S V E P$ if and only if $\mathbf{T}_{i}^{\prime}=\left(R_{1} S_{1}, \cdots, S_{i} R_{i}, \cdots\right.$, $\left.R_{n} S_{n}\right)$ has $S V E P$ for every $i \in\{1, \cdots, n\}$.

In particular, $\mathbf{R S}$ has $S V E P$ if and only if $\mathbf{S R}$ has $S V E P$.

The next lemma is crucial in our proof and is of independent interest. We will assume for convenience that $i=n$. 
Lemma 2. Let $\mathcal{U}$ be an open neighborhood of zero in $\mathbb{C}^{n}, \mathbf{T}=\left(T_{1}, \cdots, T_{n}\right)$ be a bounded commuting $n$-tuple such that $\hat{\mathbf{T}}=\left(T_{1}, \cdots, T_{n-1}\right)$ has SVEP at zero (in $\mathbb{C}^{n-1}$ ) and $M_{z_{n}}$ be the pointwise multiplication by the independent variable $z_{n}$. We have

$$
M_{z_{n}} H^{p}(\mathbf{T}-\mathbf{z}, \mathcal{O}(\mathcal{U}, X))=\{0\} \Longrightarrow H^{p}(\mathbf{T}-\mathbf{z}, \mathcal{O}(\mathcal{U}, X))=\{0\} .
$$

In particular, if $\left(T_{1}, \cdots, T_{n-1}\right)$ has $S V E P$, then the claim $M_{z_{n}} H^{p}(\mathbf{T}-\mathbf{z}, \mathcal{O}(\mathcal{U}, X))$ $=\{0\}$ yields to $H^{p}(\mathbf{T}-\mathbf{z}, \mathcal{O}(\mathcal{U}, X))=\{0\}$ for every open set $\mathcal{U}$.

Proof of Lemma 2. We first fix some notation. For $g=\sum_{I} g_{I} e_{I} \in \Lambda[\mathcal{O}(\mathcal{U}, X)]$, we denote

$$
\rho\left(g\left(z_{1}, \cdots, z_{n}\right)\right)=g\left(z_{1}, \cdots, z_{n-1}, 0\right) \text { and } \pi(g)=\rho\left(\sum_{n \notin I} g_{I} e_{I}\right) .
$$

For simplicity, we shall use the notation $\delta_{\mathbf{T}}$ for the operator $\alpha_{\mathbf{T}-\mathbf{z}}$. Let $f \in \operatorname{ker}\left(\delta_{\mathbf{T}}^{p}\right)$ be such that $z_{n} f=\delta_{\mathbf{T}}(g)$ for some $g \in \Lambda^{p-1}[\mathcal{O}(\mathcal{U}, X)]$. We have

$$
\begin{aligned}
0=\rho\left(z_{n} f\right)= & \rho\left(\sum_{n \notin I} \sum_{i=1}^{n-1}\left(T_{i}-z_{i}\right) g_{I} e_{i} \wedge e_{I}\right) \\
& +\rho\left(\sum_{n \notin I}\left(T_{n}-z_{n}\right) g_{I} e_{n} \wedge e_{I}+\sum_{n \in I} \sum_{i=1}^{n-1}\left(T_{i}-z_{i}\right) g_{I} e_{i} \wedge e_{I}\right) .
\end{aligned}
$$

Hence we have

$$
\rho\left(\sum_{n \notin I} \sum_{i=1}^{n-1}\left(T_{i}-z_{i}\right) g_{I} e_{i} \wedge e_{I}\right)=0
$$

and

$$
\rho\left(\sum_{n \notin I}\left(T_{n}-z_{n}\right) g_{I} e_{n} \wedge e_{I}+\sum_{n \in I} \sum_{i=1}^{n-1}\left(T_{i}-z_{i}\right) g_{I} e_{i} \wedge e_{I}\right)=0 .
$$

Equation (6), together with the $S V E P$ of $\hat{\mathbf{T}}=\left(T_{1}, \cdots, T_{n-1}\right)$, yields to the existence of $h=\sum_{J \subset\{1, \cdots, n-1\}} h_{J} e_{J}$ such that $\pi(g)=\delta_{\hat{\mathbf{T}}}(h)$. That is,

$$
\rho\left(\sum_{n \in I} \sum_{i=1}^{n-1}\left(T_{i}-z_{i}\right) g_{I} e_{i} \wedge e_{I}\right)=\sum_{i=1}^{n-1}\left(T_{i}-z_{i}\right) h_{J} e_{i} \wedge e_{J} .
$$

Now, equation (17) gives

$$
\begin{aligned}
0 & =\rho\left(\sum_{n \notin I}\left(T_{n}-z_{n}\right) g_{I} e_{n} \wedge e_{I}+\sum_{n \notin I} \sum_{i=1}^{n-1}\left(T_{i}-z_{i}\right) g_{I} e_{i} \wedge e_{I}\right) \\
& =e_{n} \wedge\left[T_{n} \rho\left(\sum_{n \notin I} g_{I} e_{I}\right)-(-1)^{p} \rho\left(\sum_{n \in I} \sum_{i=1}^{n-1}\left(T_{i}-z_{i}\right) g_{I} e_{i} \wedge e_{I \backslash\{n\}}\right)\right],
\end{aligned}
$$

and again using $S V E P$ of the operator $\hat{\mathbf{T}}$, we deduce that $T_{n} h-(-1)^{p} \tilde{\pi}(g)=\delta_{\hat{\mathbf{T}}}(k)$ for some $k \in \Lambda[\mathcal{O}(\mathcal{U}, X)]$. Here, we set $\tilde{\pi}(g)=\rho\left(\sum_{n \in I} g_{I} e_{I \backslash\{n\}}\right)$. Combining this last expression with the formula $\rho(g)=\pi(g)+\tilde{\pi}(g) \wedge e_{n}$, we claim that 


$$
\begin{aligned}
G=g- & \rho(g)+(-1)^{p} z_{n} h \wedge e_{n} \text { satisfies } z_{n} f=\delta_{\mathbf{T}}(G) . \text { Indeed, } \\
\delta_{\mathbf{T}}(G) & =\delta_{\mathbf{T}}(g)-\delta_{\mathbf{T}}(\rho(g))+\delta_{\mathbf{T}}\left((-1)^{p} z_{n} h \wedge e_{n}\right) \\
& =z_{n} f-\delta_{\mathbf{T}}\left[\pi(g)+\tilde{\pi}(g) \wedge e_{n}\right]+(-1)^{p} z_{n} \delta_{\hat{\mathbf{T}}} h \wedge e_{n} \\
& =z_{n} f-\delta_{\mathbf{T}} \pi(g)-\delta_{\hat{\mathbf{T}}} \tilde{\pi}(g) \wedge e_{n}+(-1)^{p} z_{n} \delta_{\hat{\mathbf{T}}} h \wedge e_{n} \\
& =z_{n} f-\left[\delta_{\hat{\mathbf{T}}}+\left(T_{n}-z_{n}\right) \otimes E_{n}\right] \delta_{\hat{\mathbf{T}}}(h)-\delta_{\hat{\mathbf{T}}} \tilde{\pi}(g) \wedge e_{n}+(-1)^{p} z_{n} \delta_{\hat{\mathbf{T}}} h \wedge e_{n} \\
& =z_{n} f-(-1)^{p-1}\left(T_{n}-z_{n}\right) \delta_{\hat{\mathbf{T}}}(h) \wedge e_{n}-\delta_{\hat{\mathbf{T}}} \tilde{\pi}(g) \wedge e_{n}+(-1)^{p} z_{n} \delta_{\hat{\mathbf{T}}} h \wedge e_{n} \\
& =z_{n} f-(-1)^{p-1}\left[T_{n} \delta_{\hat{\mathbf{T}}}(h)-(-1)^{p} \delta_{\hat{\mathbf{T}}} \tilde{\pi}(g)\right] \wedge e_{n} \\
& =z_{n} f+(-1)^{p} \delta_{\hat{\mathbf{T}}}\left[T_{n} h-(-1)^{p} \tilde{\pi}(g)\right] \wedge e_{n} \\
& =z_{n} f+(-1)^{p} \delta_{\hat{\mathbf{T}}}\left(\delta_{\hat{\mathbf{T}}} k\right) \wedge e_{n} \\
& =z_{n} f .
\end{aligned}
$$

To end the proof of the lemma, we notice that $H(z)=\frac{G(z)}{z_{n}}$ is analytic and satisfies $\delta_{\mathbf{T}}(H)=f$.

Proof of Theorem 3. Let $z \in \mathbb{C}^{n}$ be arbitrary. The componentwise actions

$$
\begin{cases}\Phi: \Lambda^{p}[\mathcal{O}(\mathcal{U}, X)] \longrightarrow \Lambda^{p}[\mathcal{O}(\mathcal{U}, X)], & u \mapsto R_{n} u \\ \Psi: \Lambda^{p}[\mathcal{O}(\mathcal{U}, X)] \longrightarrow \Lambda^{p}[\mathcal{O}(\mathcal{U}, X)], & u \mapsto S_{n} u\end{cases}
$$

induce the next morphisms of complexes

$$
\begin{gathered}
K .(\mathbf{T}-\mathbf{z}, \mathcal{O}(\mathcal{U}, X)) \stackrel{\Phi}{\longrightarrow} K .\left(\mathbf{T}^{\prime}-\mathbf{z}, \mathcal{O}(\mathcal{U}, X)\right), \\
K .\left(\mathbf{T}^{\prime}-\mathbf{z}, \mathcal{O}(\mathcal{U}, X)\right) \stackrel{\Psi}{\longrightarrow} K .(\mathbf{T}-\mathbf{z}, \mathcal{O}(\mathcal{U}, X)),
\end{gathered}
$$

since $I-\psi \phi$ is given by componentwise action with

$$
z_{n} I-S_{n} R_{n}=\sum_{v=1}^{n}\left(z_{v}-T_{v}\right) C_{v}
$$

where $C_{v}=\delta_{n, v} I \in\{\mathbf{T}\}^{\prime}$ for $v=1, \cdots, n$. It follows (Lemma 2.2.4 in [7]) that $\Psi \Phi=M_{z_{n}}$ is the pointwise multiplication by the variable $z_{n}$ on $H^{p}(\mathbf{T}-\mathbf{z}, \mathcal{O}(D, X))$.

On the other hand, from the identity $z_{n} I-R_{n} S_{n}=\sum_{v=1}^{n}\left(z_{v}-T_{v}^{\prime}\right) C_{v}$, where $C_{v}^{\prime}=\delta_{n, v} I \in\left\{\mathbf{T}^{\prime}\right\}^{\prime}$ for $v=1, \ldots, n$, it follows exactly in the same way that $\Phi \Psi=M_{z_{n}}$ on $H^{p}\left(\mathbf{T}^{\prime}-\mathbf{z}, \mathcal{O}(D, X)\right)$ for $p=0, \cdots, n$.

Thus, we have seen that

$$
\left\{\begin{array}{l}
H^{p}\left(\mathbf{T}^{\prime}-\mathbf{z}, \mathcal{O}(D, X)\right) \stackrel{\Phi}{\longrightarrow} H^{p}(\mathbf{T}-\mathbf{z}, \mathcal{O}(D, X)), \\
H^{p}(\mathbf{T}-\mathbf{z}, \mathcal{O}(D, X)) \stackrel{\Psi}{\longrightarrow} H^{p}\left(\mathbf{T}^{\prime}-\mathbf{z}, \mathcal{O}(D, X)\right)
\end{array}\right.
$$

are continuous vector-space morphisms.

Now if we assume that $H^{p}(\mathbf{T}-\mathbf{z}, \mathcal{O}(D, X))=\{0\}$, we will derive by using the previous discussion that $M_{z_{n}} H^{p}\left(\mathbf{T}^{\prime}-\mathbf{z}, \mathcal{O}(D, X)\right)=\{0\}$, and hence, from Lemma2 2 we get $H^{p}\left(\mathbf{T}^{\prime}-\mathbf{z}, \mathcal{O}(\mathcal{U}, X)\right)=\{0\}$. In particular, $\mathbf{T}$ has $S V E P$ if and only if $\mathbf{T}^{\prime}$ has $S V E P$.

Combining equation (5) with some minor modifications of the previous proof, we obtain the next $(\beta)$-version of Lemma 2. This lemma is to be used in the proof of the corresponding theorem for Bishop's property $(\beta)$. 
Lemma 3. Let $\mathcal{U}$ be an open neighborhood of zero in $\mathbb{C}^{n}$ and let $\mathbf{T}=\left(T_{1}, \cdots, T_{n}\right)$ be a bounded commuting $n$-tuple such that $\left(T_{1}, \cdots, T_{n-1}\right)$ satisfies Bishop's property $(\beta)$. Then the pointwise multiplication by the independent variable $z_{n}$ on $\Lambda^{n-1}[\mathcal{O}(\mathcal{U}, X)] / \operatorname{Im}\left(\alpha_{\mathbf{T}-\mathbf{z}}^{n-2}\right)$ is bounded below.

Theorem 4. Let $\mathbf{R}$ and $\mathbf{S}$ be commuting $n$-tuples nearly commuting. Then $\mathbf{T}=$ $\left(R_{1} S_{1}, \cdots, R_{i} S_{i}, \cdots, R_{n} S_{n}\right)$ has $(\beta)$ if and only if $\mathbf{T}^{\prime}{ }_{i}=\left(R_{1} S_{1}, \cdots, S_{i} R_{i}, \cdots, R_{n} S_{n}\right)$ has $(\beta)$ for every $i \in\{1, \cdots, n\}$.

In particular, $\mathbf{R S}$ has $(\beta)$ if and only if $\mathbf{S R}$ has $(\beta)$.

Proof. As for $S V E P$, we will take $i=n$, and since $(\beta)$ implies $S V E P$, we only need to show the last step. That is, the pointwise multiplication by the independent variable $z_{n}$ on $\Lambda^{n-1}[\mathcal{O}(\mathcal{U}, X)] / \operatorname{Im}\left(\alpha_{T}^{n-2}\right)$ is bounded below if and only if the corresponding mapping on $\Lambda^{n-1}[\mathcal{O}(\mathcal{U}, X)] / \operatorname{Im}\left(\alpha_{\mathbf{T}^{\prime}-\mathbf{z}}^{n-2}\right)$ is bounded below. Assume that $\alpha_{\mathbf{T}^{\prime}-\mathbf{z}}\left(f_{p}\right)$ converges to zero. It will be immediate that $\lim _{n \rightarrow \infty} \alpha_{\mathbf{T}-\mathbf{z}}\left(S_{n} f_{p}\right)=0$. Now the fact that $\Lambda^{n-1}[\mathcal{O}(\mathcal{U}, X)] / \operatorname{Im}\left(\alpha_{T}^{n-2}\right)$ is bounded below yields to the existence of $g_{p}$ such that $S_{n} f_{p}-\alpha_{\mathbf{T}-\mathbf{z}} g_{p}$ tends to zero. We apply $R_{n}$, and we get

$$
\lim _{n \rightarrow \infty}\left(R_{n} S_{n} f_{p}-\alpha_{\mathbf{T}^{\prime}-\mathbf{z}}\left(R_{n} g_{p}\right)\right)=0 .
$$

Equivalently,

$$
\lim _{n \rightarrow \infty}\left(z_{n} f_{p}+\left(R_{n} S_{n}-z_{n}\right) f_{p}-\alpha_{\mathbf{T}^{\prime}-\mathbf{z}}\left(R_{n} g_{p}\right)\right)=0 .
$$

From the Kronecker formula $\left(R_{n} S_{n}-z_{n}\right)=E_{n}^{*} \alpha_{\mathbf{T}^{\prime}-\mathbf{z}}+\alpha_{\mathbf{T}^{\prime}-\mathbf{z}} E_{n}^{*}$, we deduce that

$$
\lim _{n \rightarrow \infty}\left(z_{n} f_{p}+\left(E_{n}^{*} \alpha_{\mathbf{T}^{\prime}-\mathbf{z}}+\alpha_{\mathbf{T}^{\prime}-\mathbf{z}} E_{n}^{*}\right) f_{p}-\alpha_{\mathbf{T}^{\prime}-\mathbf{z}}\left(R_{n} g_{p}\right)\right)=0
$$

and then

$$
\lim _{n \rightarrow \infty}\left(z_{n} f_{p}+\alpha_{\mathbf{T}^{\prime}-\mathbf{z}}\left[E_{n}^{*} f_{p}-R_{n} g_{p}\right]\right)=0 .
$$

We end the proof of our theorem by using Lemma 3 .

4.2. The case of criss-cross commutativity. In the setting of criss-cross commutativity, we have

Theorem 5. Let $\mathbf{R}$ and $\mathbf{S}$ be commuting n-tuples satisfying the criss-cross commuting. Then,

RS has SVEP (resp. has ( $\beta)$ ) if and only if $\mathbf{S R}$ has SVEP (resp. has ( $\beta)$ ).

Proof. For $n=1$, the result can be found in [3, Proposition 2.1].

For $n=2$, the $S V E P$ of $\left(R_{1} S_{1}, R_{2} S_{2}\right)$ reads as follows:

$$
\left\{\begin{array}{l}
\operatorname{ker}\left(R_{1} S_{1}-z_{1}\right) \cap \operatorname{ker}\left(R_{2} S_{2}-z_{2}\right)=\{0\}, \\
\left\{\left(f_{1}, f_{2}\right):\left(R_{1} S_{1}-z_{1}\right) f_{2}=\left(R_{2} S_{2}-z_{2}\right) f_{1}\right\} \\
\quad=\left\{\left(\left(R_{1} S_{1}-z_{1}\right) g,\left(R_{2} S_{2}-z_{2}\right) g\right): g \in \mathcal{O}(D, X)\right\} .
\end{array}\right.
$$

Assume that $\left(R_{1} S_{1}, R_{2} S_{2}\right)$ has $S V E P$ and let us show that

$$
\left\{\begin{array}{l}
\operatorname{ker}\left(S_{1} R_{1}-z_{1}\right) \cap \operatorname{ker}\left(S_{2} R_{2}-z_{2}\right)=\{0\}, \\
\left\{\left(f_{1}, f_{2}\right):\left(S_{1} R_{1}-z_{1}\right) f_{2}=\left(S_{2} R_{2}-z_{2}\right) f_{1}\right\} \\
\quad=\left\{\left(\left(S_{1} R_{1}-z_{1}\right) g,\left(S_{2} R_{2}-z_{2}\right) g\right): g \in \mathcal{O}(D, X)\right\} .
\end{array}\right.
$$

Consider $f$ satisfying

$$
\left(S_{1} R_{1}-z_{1}\right) f=\left(S_{2} R_{2}-z_{2}\right) f=0
$$


and apply $R_{1}$ to the latter equality. We will get

$$
\left(R_{1} S_{1}-z_{1}\right) R_{1} f=\left(R_{2} S_{2}-z_{2}\right) R_{1} f=0 .
$$

From equation (9), it follows that $R_{1} f \equiv 0$. Now from equation (11), we have $z_{1} f \equiv 0$ and hence $f \equiv 0$.

For the second affirmation, let $\left(f_{1}, f_{2}\right)$ be such that $\left(S_{1} R_{1}-z_{1}\right) f_{2}=\left(S_{2} R_{2}-\right.$ $\left.z_{2}\right) f_{1}$. Applying $R_{1}$ gives $\left(R_{1} S_{1}-z_{1}\right) R_{1} f_{2}=\left(R_{2} S_{2}-z_{2}\right) R_{1} f_{1}$. The $S V E P$ of $\left(R_{1} S_{1}, R_{2} S_{2}\right)$ implies that there exists $g \in \mathcal{O}(D, X)$ satisfying $R_{1} f_{1}=\left(R_{1} S_{1}-z_{1}\right) g$ and $R_{1} f_{2}=\left(R_{2} S_{2}-z_{2}\right) g$. We derive that $S_{1} R_{1} f_{1}=\left(S_{1} R_{1}-z_{1}\right) S_{1} g$ and $S_{1} R_{1} f_{2}=$ $\left(S_{2} R_{2}-z_{2}\right) S_{1} g$. The latter expression gives

$$
z_{1} f_{1}=\left(S_{1} R_{1}-z_{1}\right)\left(S_{1} g-f_{1}\right) \text { and } z_{1} f_{2}=\left(S_{2} R_{2}-z_{2}\right)\left(S_{1} g-f_{1}\right) .
$$

It now suffices to establish that $\frac{1}{z_{1}}\left(S_{1} g-f_{1}\right) \in \mathcal{O}(D, X)$. To this aim, if we take $z_{1}=0$ we obtain $0=\left(S_{2} R_{2}-z_{2}\right)\left(S_{1} g\left(0, z_{2}\right)-f_{1}\left(0, z_{2}\right)\right)$. Using the $S V E P$ of $S_{2} R_{2}$, we deduce that $S_{1} g\left(0, z_{2}\right)-f_{1}\left(0, z_{2}\right) \equiv 0$. Finally, $\frac{1}{z_{1}}\left(S_{1} g-f_{1}\right) \in \mathcal{O}(D, X)$.

In an inductive proof, assume now that we have showed our claim for $n-1$ and let $\mathbf{T}=\left(R_{1} S_{1}, \cdots, R_{n} S_{n}\right)$ be with $S V E P$. In particular, $\left(R_{1} S_{1}, \cdots, R_{n-1} S_{n-1}\right)$ will satisfy $S V E P$ and hence the same is the case for $\left(S_{1} R_{1}, \cdots, S_{n-1} R_{n-1}\right)$. Now, if $H^{p}\left(\left(R_{1} S_{1}, \cdots, R_{n} S_{n}\right)\right)=0$, using the same morphisms as in Lemma 2 we get $M_{z_{n}} H^{p}\left(\left(S_{1} R_{1}, \cdots, S_{n} R_{n}\right)\right)=\{0\}$. Then $\left(S_{1} R_{1}, \cdots, S_{n} R_{n}\right)$ has $S V E P$.

The proof for Bishop's property $(\beta)$ is handled in the same way as for the near commutativity condition.

\section{LOCAL SPECTRA FOR RS AND SR}

We first recall the definition of local spectrum for $n$-tuples. Let $\mathbf{T}=\left(T_{1}, \cdots, T_{n}\right)$ denote a commuting $n$-tuple of bounded linear operators acting on the Banach space $X$. For $x \in X, \rho(\mathbf{T}, x)$ is the set of those points $\lambda=\left(\lambda_{1}, \cdots, \lambda_{n}\right) \in \mathbb{C}^{n}$ for which there exist an open set $U \ni \lambda$ and $X$-valued analytic functions on $U: f_{1}, \cdots, f_{n}$ such that

$$
\sum_{j=1}^{n}\left(T_{j}-z_{j}\right) f_{j}(z)=x, \quad z \in U .
$$

The set $\sigma(\mathbf{T}, x))=: \mathbb{C}^{n} \backslash \rho(\mathbf{T}, x)$ is called the analytic local spectrum of $\mathbf{T}$ at $x$.

We have the following result, which generalizes the one in the single operator case.

Proposition 3. Let $\mathbf{R}$ and $\mathbf{S}$ be commuting $n$-tuples and let $x \in X$. We have:

(1) If $\mathbf{R}$ and $\mathbf{S}$ are nearly commuting (3), then

$$
\sigma\left(\mathbf{S R}, S_{i} x\right) \subset \sigma\left(\mathbf{S R}_{\{i\}}, x\right) \subset \sigma\left(\mathbf{S R}, S_{i} x\right) \cup[0]^{\{i\}} .
$$

(2) If $\mathbf{R}$ and $\mathbf{S}$ are criss-cross commuting, then

$$
\sigma\left(\mathbf{S R}, S_{i} x\right) \subset \sigma(\mathbf{R S}, x) \subset \bigcap_{j=1}^{n} \sigma\left(\mathbf{S R}, S_{j} x\right) \cup[0]^{\{j\}} .
$$

Proof. (1) For simplicity, we will assume that $i=1$. Let $\lambda \notin \sigma\left(\mathbf{S R}_{\{1\}}, x\right)$ and $f_{1}, \cdots, f_{n}$ be $X$-valued analytic functions in a neighborhood $\mathcal{U}$ of $\lambda$ such that 
$x=\left(R_{1} S_{1}-z_{1}\right) f_{1}(z)+\sum_{j=2}^{n}\left(S_{j} R_{j}-z_{j}\right) f_{j}(z)$ for every $z \in \mathcal{U}$. Then $S_{1} x=\left(S_{1} R_{1}-\right.$ $\left.z_{1}\right) S_{1} f_{1}(z)+\sum_{j=2}^{n}\left(S_{j} R_{j}-z_{j}\right) S_{1} f_{j}(z)$. Since $S_{1} f_{j}$ are $X$-valued analytic functions in a neighborhood $U$ of $\lambda$, we derive that $\lambda \notin \sigma\left(\mathbf{S R}, S_{1} x\right)$.

For the other inclusion, let $\lambda \notin \sigma\left(\mathbf{S R}_{\{1\}}, S_{1} x\right) \cup[0]^{\{1\}}$ and $f_{1}, \cdots, f_{n}$ be $X$-valued analytic functions in a neighborhood $\mathcal{U}$ of $\lambda$ such that $S_{1} x=\left(S_{1} R_{1}-z_{1}\right) f_{1}(z)+$ $\sum_{j=2}^{n}\left(S_{j} R_{j}-z_{j}\right) f_{j}(z)$ for every $z \in \mathcal{U}$. Then by applying $R_{1}, S_{1}\left[-x+R_{1} f_{1}\right]+$ $\sum_{j=2}^{n}\left(S_{j} R_{j}-z_{j}\right) f_{j}=z_{1} f_{1}$. Since $\lambda \notin[0]^{\{1\}}$, we may choose $\mathcal{U}$ such that $z_{1} \neq 0$ for every $z \in \mathcal{U}$. Now consider $g_{1}=\frac{1}{z_{1}}\left[-x+R_{1} f_{1}\right]$ and $g_{j}=\frac{1}{z_{1}} R_{1} f_{j}$ for $j=2, \cdots, n$. Then a simple and direct calculation gives

$$
\begin{aligned}
\left(R_{1} S_{1}-z_{1}\right) g_{1}+\sum_{j=2}^{n}\left(S_{j} R_{j}-z_{j}\right) g_{j}= & \frac{1}{z_{1}}\left[R_{1}\left(S_{1} R_{1}-z_{1}\right) f_{1}-R_{1} S_{1} x+z_{1} x\right] \\
& +\frac{1}{z_{1}} R_{1}\left[\sum_{j=2}^{n}\left(S_{j} R_{j}-z_{j}\right) f_{j}\right] \\
& =\frac{1}{z_{1}}\left[R_{1}\left(\sum_{j=1}^{n}\left(S_{j} R_{j}-z_{j}\right) f_{j}\right)-R_{1} S_{1} x+z_{1} x\right] \\
& =x .
\end{aligned}
$$

Thus, $\lambda \notin \sigma(\mathbf{S R}, x)$.

(2) The second statement is obtained similarly.

\section{REFERENCES}

1. E. Albrecht and J. Eschmeier, Analytic functional models and local spectral theory. Proc. London Math. Soc. 75 (1997) 323-348. MR1455859 (98f:47043)

2. B. Barnes, Common operator properties of the linear operators $R S$ and $S R$. Proc. Amer. Math. Soc. 126 (1998) 1055-1061. MR1443814 (98f:47003)

3. C. Benhida and E. H. Zerouali, Local spectral theory of linear operators RS and SR. Integral Equations Operator Theory 54 (2006) 1-8. MR2195227(2006i:47009)

4. C. Benhida and E. H. Zerouali, On Taylor and other joint spectra for commuting $n$-tuples of operators. J. Math. Anal. Appl. 326 (2007) 521-532. MR2277800|(2007h:47012)

5. M. Chō, R. E. Curto, and T. Huruya, n-tuples of operators satisfying $\sigma_{T}(A B)=\sigma_{T}(B A)$. Special issue dedicated to Professor T. Ando. Linear Algebra Appl. 341 (2002) 291-298. MR $1873626(2002 \mathrm{k}: 47014)$

6. J. Eschmeier, Local properties of Taylor's analytic functional calculus. Invent. Math. 68 (1982) 103-116. MR666637 (83m:47017)

7. J. Eschmeier and M. Putinar, Spectral Decompositions and Analytic Sheaves. London Mathematical Society Monographs, New Series, 10. Oxford University Press, 1996. MR:1420618 (98h:47002)

8. J. K. Finch, The single valued extension property on a Banach space. Pacific J. Math. 58 (1975) 61-69. MR0374985 (51:11181)

9. R. Harte, On criss-cross commutativity. J. Operator Theory 37 (1997) 303-309. MR1452279 (98e:47004)

10. R. Harte, On criss-cross commutativity. II. J. Operator Theory 46 (2001) 39-43. MR:1862178 (2002k:47011)

11. S. Li, Taylor spectral invariance for crisscross commuting pairs on Banach spaces. Proc. Amer. Math. Soc. 124 (1996) 2069-2071. MR1322934 (96i:47007) 
12. M. Putinar, Some recent developments in operator theory. CBMS Regional Conference Series in Mathematics, No. 36, Amer. Math. Soc., Providence, RI, 1978. MR0487495 (58:7120)

13. J. L. Taylor, A joint spectrum for several commuting operators. J. Funct. Anal. 6 (1970) 172-191. MR0268706 (42:3603)

14. V. Muller, Spectral theory of linear operators. Operator Theory Adv. Appl. 187 (2008).

15. F-H. Vasilescu, On pairs of commuting operators. Studia Math. 62 (1978) 203-207. MR 0493434 (58:12441)

16. F-H. Vasilescu, A characterization of the joint spectrum in Hilbert spaces. Rev. Roum. Math. Pures Appl. 22 (1977) 1003-1009. MR0500211 (58:17890)

17. F-H. Vasilescu, Analytic functional calculus and spectral decompositions. Editura Academiei Republicii Socialiste Romania, Bucharest, 1982. MR690957 (85b:47016)

UFR de Mathématiques - CNRS-UMR 8524, Université de Lille 1, BÂt M2, 59655 Villeuneuve CEDex, France

E-mail address: benhida@math.univ-lille1.fr

Faculté des Sciences de Rabat, Université Mohammed V, BP 1014, Rabat, Morocco

E-mail address: zerouali@fsr.ac.ma 